\title{
Synthesis, characterization and spectral studies of various newer long chain aliphatic Acid (2-hydroxy benzylidene and 1H-indol-3-ylmethylene) hydrazides as mosquito para-pheromones
}

\author{
Suman Awasthi*, Poonam Rishishwar, Ambati N. Rao, Kumaran Ganesan, \\ and Ramesh Chandra Malhotra \\ Sinthetic Chemistry Division, Defence Reseath \& Development Estoblishment, Jhansi Road, Gwalior 474002 India \\ (2007.4.3 접수)
}

\section{Synthesis, characterization and spectral studies of various newer long chain aliphatic acid (2-hydroxy benzylidene and $1 \mathrm{H}$-indol-3-ylmethylene) hydrazides as mosquito para-pheromones}

\author{
Suman Awasthi*, Poonam Rishishwar, Ambati N. Rao, Kumaran Ganesan, \\ and Ramesh Chandra Malhotra \\ Sinthetic Chemistry Division, Defence Research de Development Establishment, Jhansi Road, Gualior-474102 India \\ (Received April 3, 2007)
}

8 욕. 여러가지 긴 사슬 지방산 하이드라지드는 방햠족과 헤테로 고러 알데히드와 반응하여 알코올 용매하에서 2hydroxy benzylidene와 새로운 모기 파라-폐로론인1H-indol-3-ylmethylene hydrazides을 얻었다. 마이크로파의 조사기 술과 마찬가지로 전통적인 방법에 의한 다양한 새로운 긴고러 지방산 하이드라지드 (2-hydroxy benzylidene와 $1 \mathrm{H}$ indol-3-ylmethylene)의 합성방법도 보고한다. 이 화합물들의 구조는 FTIR, NMR \& MS와 같은 분광학적 기법에 의해 증명되었다. 이 화합물의 전자훔격질량스켁트럼 분쇄 패턴의 몇 가지 채미있는 특징도 논의했다.

주제어: 긴 사슬 지방산 하이드라지드, 긴 사슬 지방산(2-hydroxy benzylidene\& $1 \mathrm{H}$-indol-3-ylmethylene) 하이드라 지드, 로기 파라-페로몬, 마이크로파

\begin{abstract}
Various long chain aliphatic acid hydrazides react with aromatic and heterocyclic aldelydes in alcoholic medium in refluxing conditions to give corresponding 2-hydroxy benzylidene and $1 \mathrm{H}$-indol-3-ylmethylene hydrazides, a newer class of mosquito para-pheromones. We describe here synthesis of various novel long chain aliphatic acid (2hydroxy benzylidene and $1 \mathrm{H}$-indol-3-ylmethylene) hydrazides by conventional as well as microwave iradiation techniques. The stuctures of these compounds have been confumed by spectroscopic tecluiques (FTIR, NMR \& MS). Some of the interesting features of the electron impact mass spectral fragmentation pattern of these compounds have also been discussed. Keywords: Long Chain Aliphatic Acid Hydrazide. Long Chain Aliphatic Acid (2-hydroxy benzylidene \& 1H-indol-3ylmethylene) Hydrazide, Mosquito Para-pheromones, Microwave.
\end{abstract}

\section{INTRODLCTION}

Acyl derivatives of hydrazine are called acid hydrazides. They constitute an important class of biologically active organic compounds. Hydrazides and their condensation products are reported to posses a wide range of antibacterial activity ${ }^{\text {l.2 }}$ and tuberculostatic $\mathrm{c}^{3-1}$ properties. Similarly derivatives of aryloxyacetyl hydrazides have antituberculotic activities and some are used as post-emergence, selective 
herbicides ${ }^{5}$ to control terrestrial and aquatic broad leaved weeds in some plants. Another classical application of hydrazines and hydrazides is in the synthesis of heterocycles, and many heterocycles are precursors of drugs, agrochemicals and dye stuffs and are therefore of commercial importance. Some hydrazines were reported as newer class of insecticidal insect growth regulators. Tebufenozide, methoxyfenozide and chromafenozide are commercialized member of the novel group of diacylhytrazine insect growth regulators. ${ }^{6}$ From the synthetic point of view, hydrazones are important synthones for several transformations and their synthesis from various precursors is well documented. ${ }^{7}$ Various long chain acids and esters are reported as semiochemicals of egg origin that affect the ovipositional behavior of the mosquito Aedes aegupt ${ }^{8}$, which is a vector of dengue, chikungunya and yellow fever. Similarly, substantial documentation is available for oviposition-stimulating semiochemicals such as nheneicosane," trimethylphenol, 4-methylphenol, ${ }^{10}$ 3methylindole. ${ }^{11}$

Over the last few years, there has been growing interest in the synthesis of organic compounds under gren chenistry such as nictowave irtadiation because of increasing environmental consciousness. The feasibility of microwave assisted synthesis has been demonstrated in various transformations like condensation, ${ }^{\text {lI }}$ cycloaddition, ${ }^{\text {L" }}$ alkylation, ${ }^{\text {Lt }}$ synthesis of various heterocyclic compounds ${ }^{15-17}$ and in many other chemical reactions. The salient features of these transfornations are the enhanced reaction rate, greater selectivity and the experimental ease of manipulation ${ }^{18}$ leading to an efficient, envirommental friendly \& cost effective pathway of several syn-
Table 1. Synthesis of sarious long chain aliphatic acid hydrazides

\begin{tabular}{ccccc}
\hline \hline \multirow{2}{*}{ Product } & \multicolumn{2}{c}{ Time } & \multicolumn{3}{c}{ Yield of the product ("\%)" } \\
\cline { 2 - 5 } & $\begin{array}{c}\text { Conientional } \\
\text { (hr) }\end{array}$ & $\begin{array}{c}\text { MW } \\
\text { (min) }\end{array}$ & Conventional & MW \\
\hline $\mathbf{2 a}$ & 4 & 9 & 65 & 85 \\
$\mathbf{2 b}$ & 4 & 10 & 55 & 87 \\
$\mathbf{2 c}$ & 4 & 10 & 65 & 85 \\
\hline
\end{tabular}

${ }^{a}$ isolated product

thetically useful compounds. Keeping in view of the biological and synthetic utility of hydrazides, long chain aliphatic esters and acids, we have synthesized newer long chain acid hydrazides (2a-c, Table 1) and corresponding substituted hydrazides (3a-f, Table 3) using nicrowave irradiation technique as well as by conventional method (Scheme 1 \& 2). The synthesis of these substituted hydrazides assunies importance because of its utility from social perspective. All the above conpounds were characterized by spectral analysis.

\section{RESLLTS AND DISCLSSION}

Hydrazinolysis of various carboxylic acid hydrazides $\left[\mathrm{CH}_{3}-\left(\mathrm{CH}_{2}\right)_{n}-\mathrm{CO}-\mathrm{NH}-\mathrm{NH}_{2}, \mathrm{n}=10,12,14\right)$ from corresponding nethyl ester was carried out by conventional method as well as by nicrowave technique. The conventional method involved the refluxing of methyl ester of carboxylic acid with excess of hydrazine hydrate in ethanol nediun for 3-4 hours while in case of the nicrowave tecluique, a mixture of ester and hydrazine hydrate (1:1 molar ratio) was irradiated with microwave for 10-15 minutes without any solvent, catalyst or solid support. The

Table 2. Physical \& spectral data for compound 2b-c

\begin{tabular}{|c|c|c|c|c|}
\hline Comp & $\mathrm{mp}\left({ }^{\circ} \mathrm{C}\right)$ & Mol. formula & $\delta_{k} \cdot(J$ in $\mathrm{Hz})$ & $\mathrm{MS}\left(\mathrm{M}^{-}\right)$ \\
\hline $2 a^{+}$ & $103-105$ & $\mathrm{C}_{12} \mathrm{H}_{2} \mathrm{~N}_{2} \mathrm{O}$ & $\begin{array}{l}0.9(\mathrm{t}, 3 \mathrm{H}, J, 7.0), 1.2\left(\text { conglomerate, } 16 \mathrm{H}, 8 \mathrm{CH}_{2}\right), 2.1\left(\mathrm{p}, 2 \mathrm{H}, \mathrm{CH}_{2} J .7 .0\right), \\
2.8(\mathrm{t}, 2 \mathrm{H}, \mathrm{CH}, \mathrm{CO}, J, 6.0), 3.9\left(\mathrm{bs}, 2 \mathrm{H}, \mathrm{NH}_{2}\right), 6.8(\mathrm{~s}, 1 \mathrm{H}, \mathrm{NHCO}, \text { exclangeable) }\end{array}$ & 214 \\
\hline $2 b^{+}$ & $108 \cdot 110$ & $\mathrm{C}_{1-} \mathrm{H}_{3} \mathrm{~N}_{2} \mathrm{O}$ & $\begin{array}{l}1.0 \mathrm{t}, 3 \mathrm{H}, J, 7.0), 1.4(\mathrm{conglomerate}, 20 \mathrm{H}, 10 \mathrm{CH}), 1.9\left(\mathrm{p}, 2 \mathrm{H}, \mathrm{CH}_{-}, J, 7.0\right), 2.3(\mathrm{t}, \\
\left.2 \mathrm{H}, \mathrm{CH}_{2} \mathrm{CO}, J, 6.0\right), 3.7\left(\mathrm{bs}, 2 \mathrm{H}, \mathrm{NH}_{2}\right), 6.9(\mathrm{~s}, 1 \mathrm{H}, \mathrm{NHCO}, \text { exchangeable })\end{array}$ & 242 \\
\hline $2 c^{+}$ & $112-114$ & $\mathrm{C}_{k} \mathrm{H}_{3-1} \mathrm{~N}_{2} \mathrm{O}$ & $\begin{array}{l}0.9(\mathrm{t}, 3 \mathrm{H}, J, 7.0), 1.3\left(\text { conglomerate, } 24 \mathrm{H}, 12 \mathrm{CH} \mathrm{H}_{2}\right), 2.0\left(\mathrm{p}, 2 \mathrm{H}, \mathrm{CH}_{\mathrm{s}}, J, 7.0\right) \\
2.5\left(\mathrm{t}, 2 \mathrm{H}, \mathrm{CH} \mathrm{CH}_{2} \mathrm{CO}, J, 6.0\right), 3.8\left(\mathrm{ks}, 2 \mathrm{H}, \mathrm{NH}_{2}\right), 6.7\end{array}$ & 270 \\
\hline
\end{tabular}

2007. Fol. 5 . *io. 6 
Toble 3. Synthesis of various longy clain aliphatic acid (2-hydroxy hen/s lidene) hydravides \& (1 1 1-indol-3-y.lnethylene) hydrayides

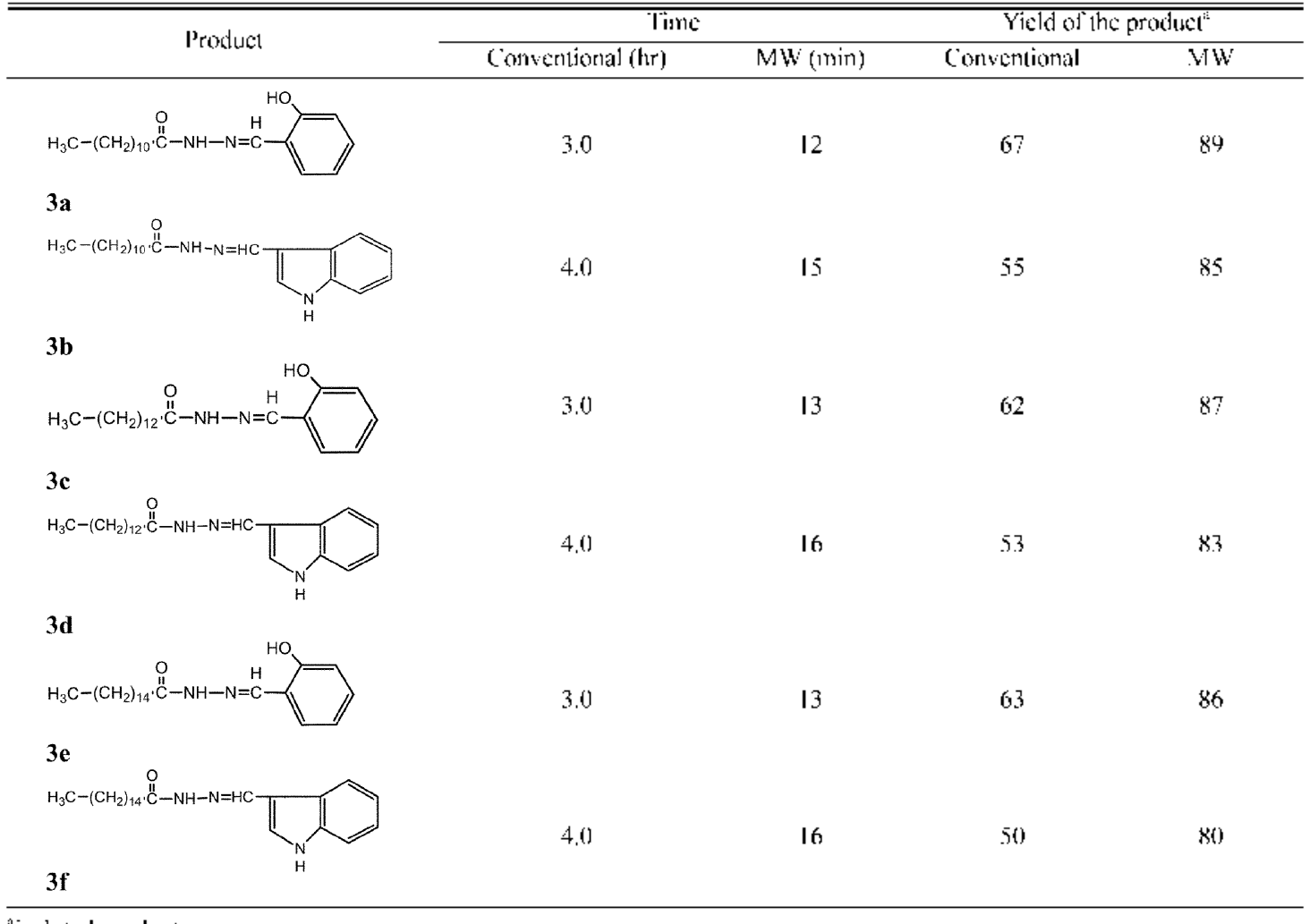

"isolated product

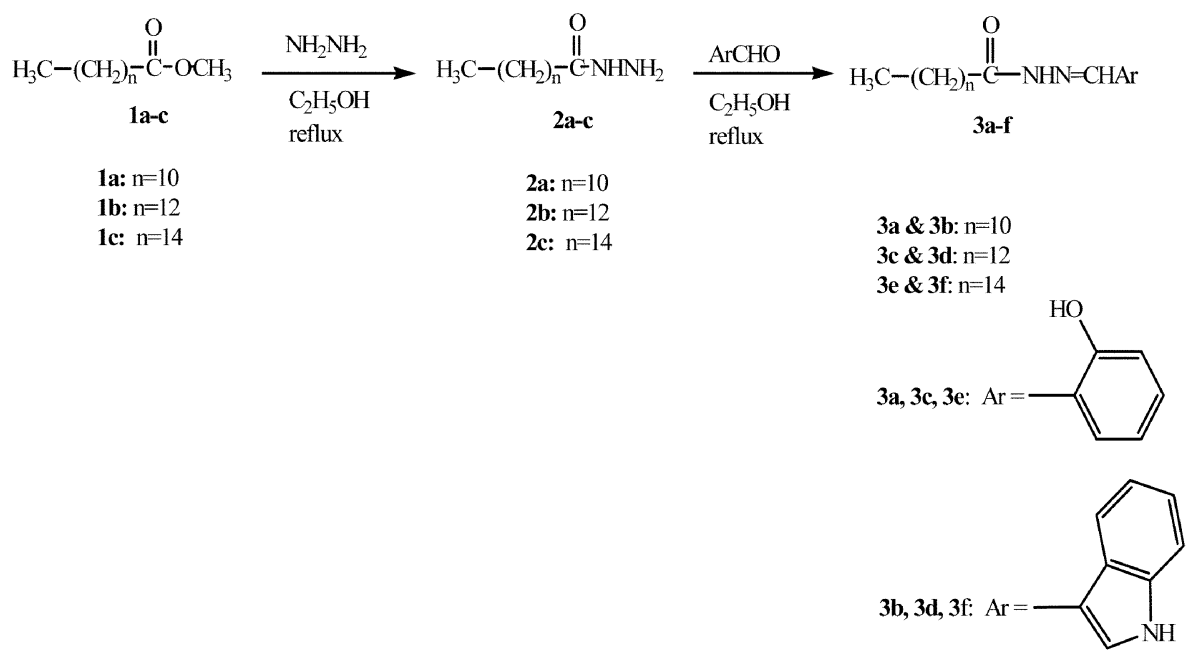

S.home I. Comventional synhesis of acid hydrazide.

results obtained from two approaches are sunmarized in Table l (Entries 2a-c).

Various newer carboxylic acid (2-hydoxybezylidene) hydrazide (3a, 3c, 3e) and carboxylic acid (1/l- indol-3-ylmethylene) hydrazide (3b, 3d, 3f) were also prepared by condensation of $2 \mathrm{a}-\mathrm{c}$ with 2-hydroxy benzaldehyde and indole-3-carboxaldehyde respectively under conventional as well as microwave 


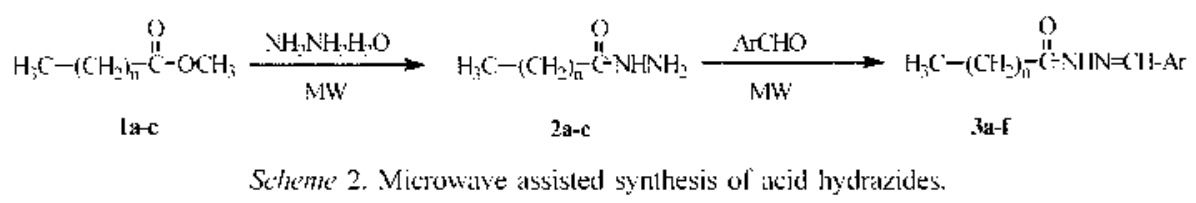

irradiation methods. In conventional method, they were prepared by refluxing $\mathbf{1}$ a-c with different aldehydes in ethanol and in microwave irradiation technique; they were prepared without using any solvent. The overall yield of the product using microwave irradiation technique was $85-90 \%$ as compared to the conventional methods in which the yields were $30-55 \%$ and reaction time was $3-10$ min by microwave irradiation as compared to 4-5 hour in conventional method.

\section{Spectral Analysis}

IR spectrum of dodecanoic acid hydrazide (2a) showed absorption band at 1635 and $1500 \mathrm{~cm}^{-1}$ corresponding to $\mathrm{C}-\mathrm{O}$ and $\mathrm{C}-\mathrm{N}$ stretching vibrations. Two bands at 3350 and $3310 \mathrm{~cm}^{-1}$ appeared due to the presence of $\mathrm{NH}_{2}$ and $\mathrm{NH}$ groups respectively. The corresponding dodecanoic acid (2-hydroxybenzyldene) hydrazide showed similar spectra except that bands due to the $\mathrm{NI}_{2}$ group. it showed band at 1575-1565 $\mathrm{cm}^{-1}$ due to the $\mathrm{C}-\mathrm{N}$ stretching vibrations. The '] ] NMR spectrum of dodecanoic acid hydrazide displayed a broad singlet at $4.2(2 \mathrm{H})$ corresponding to $\mathrm{NH}_{2}$ protons. Methyl proton $\mathrm{CH}_{3}$ displayed a triplet at 0.9 in its ' $\mathrm{H}$ NMR spectrum while a singlet at $13.8 \mathrm{ppm}$ in its ' $\mathrm{C}$. N.MR spectrum. A singlet appeared at $\delta 8.2 \mathrm{ppm}$ due to the $\mathrm{NHCO}$ proton. Presence of later was further supported by the appearance of a peak at 171.80 in its ${ }^{13} \mathrm{C}$ NMR spectrum. $\mathrm{ClI}_{2}-\mathrm{CO}$ proton displayed a triplet at 2.5 in its 'II NMR while a singlet appeared at $39.3 \mathrm{ppm}$ in its "' C NMR. A distorted quintet was appeared at 1.4 in ${ }^{1}$ l N NMR. The remaining $\mathrm{Cl}_{2}$ groups, which were very similar in chemical shift, were strongly coupled to one another; they appeared as partially resolved band and act as a conglomerate of spins in coupling to the $\mathrm{CH}_{3}$ group \& signal appeared at 1.2 in its 'H NMR. Sinilar pattern for the proton resonance was also observed in the 'H N.MR spectrum of substituted hydrazides (hydrazones). The corre- sponding substituted hydrazides (hydrazones) of $2 \mathrm{a}$ containing phenolic moiety (3a) displayed singlet corresponding $\mathrm{N}-\mathrm{CH}$ proton at $\delta 8.0-8.4$ while four benzenoid protons appeared as multiplet at 6.9-7.5 ppm. Similar pattern lor the resonance was observed in the 'II NMR spectrum ol substituted hydrazide (3b) having indolic moiety and displayed a singlet at 10.1 resonated due to indole NII protion. Four benzenoid protons and one indolic Cll proton appeared as multiplet at 7.1-7.7 ppm. In the EI mass spectra of dodecanoic acid hydrazide (2a), molecular ion pcak was weak but usually discernible and for the corresponding substituted hydrazides (hydrazones) $3 a \& 3 b$, molecular ion peaks were distinct in the mass spectra. Fragment ion peak at $m / z \quad 183$ could be attributed to the loss of $\mathrm{NHNH}_{2}$ and $\mathrm{NIIN}=\mathrm{C}] \mathrm{I}-\mathrm{\Lambda}$ radicals from the molecular ions of dodecanoic acid hydrazide (2a) and dodecanoic acid (2-hydroxybenzylidene) hydrazide (3a) \& dodecanoic acid (111 indol-3-ylmethylene) hydrazide (3b) respectively. The formation of fragnent ion at $m / z 74$ can be rationalized by the loss of neutral molecule 1-decene from molecular ion (2a) as depicted in Scheme 3. The base peak at $m / z 57$ could arise duc to the loss of methyl radical and $\mathrm{H}$. molecule from the fragment ion having $m / z$ value of 74 (Scheme 3). The fragment ion peak at $m / z 199$ could be attributed to the loss of $\mathrm{CH}_{3}$ radical from the molecular ion of dodecanoic acid hydrazide (2a). The ions corresponding $10 \mathrm{~m} / \mathrm{L} 185,171,157$, $143,129,115,101,87,73$ were formed due to loss of $\mathrm{Cl}_{2}$ group successively. In addition, Mc-Lalferty rearangement in molecular ion of 3 a gave rise to peak at $m / z 178$ and peaks at $m / z, 163,135,107$ were due to sucecssive loss of $\mathrm{CH}_{3}$ radical, $\mathrm{CO} \&$ $\mathrm{N}_{2}$ molccules. Similarly the formation of fragment ion at $m / z 20 \mathrm{l}$ can be rationalized by the loss of neutral molecule dodecene from molecular ion $\mathbf{3 b}$ which in turn gave rise to peaks at $186 \& 158$ due to subsequent loss of $\mathrm{CH}_{3}$ radical and $\mathrm{CO}$ mole- 


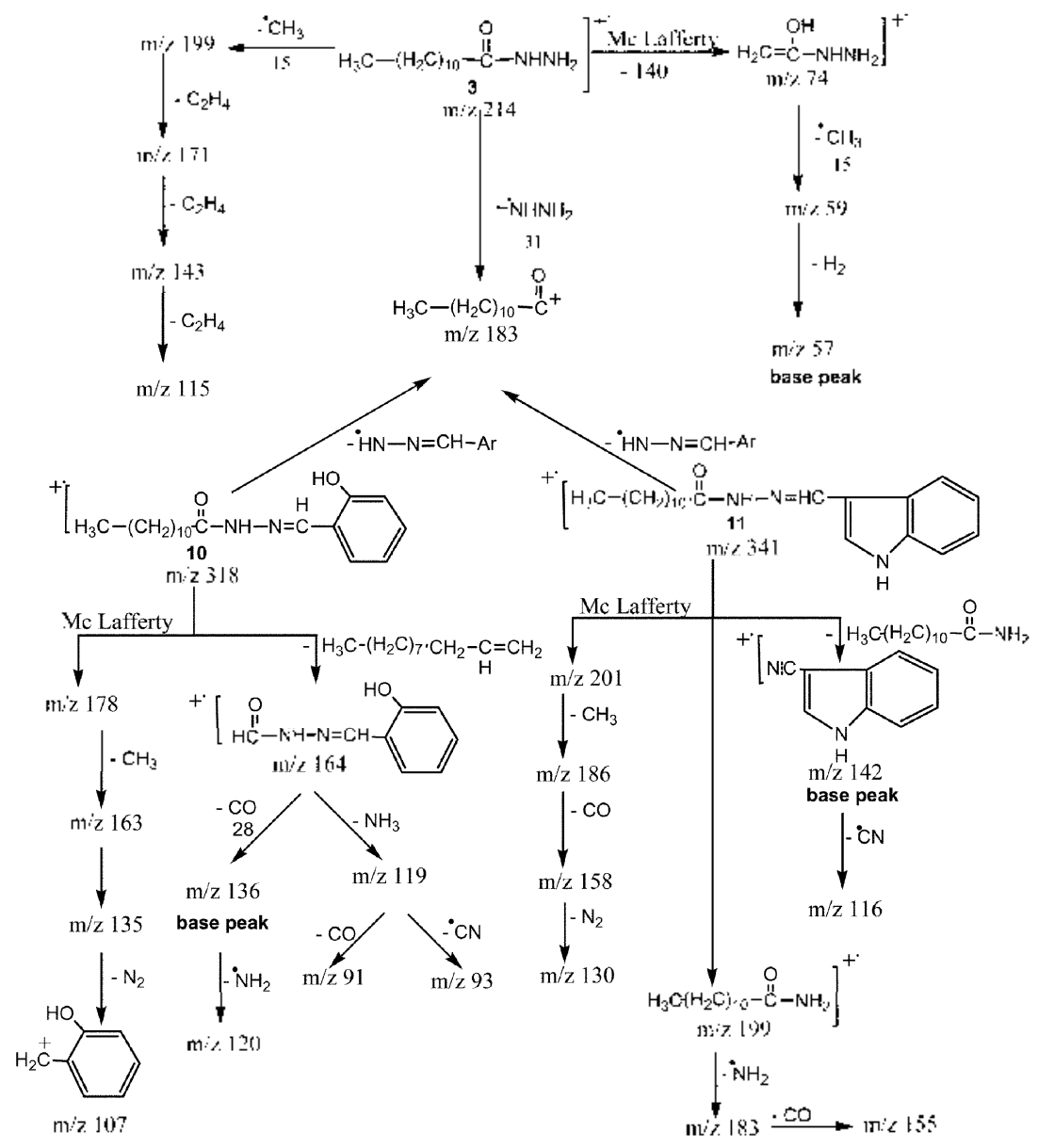

Schoni 3. Analysis of mats spectra of substituted acid hydrazides (hydrazones).

cule. The base peak at $m / 2142$ could arise due to the loss of neutral molecule tetradeconoic acid anide from molecular ion $\mathbf{3 b}$.

\section{CONCLUSION}

In sumblary, we described the microwave assisted synthesis of long chain acid hydrazides and various newer cortesponding benzylidene hydrazides \& indolylmethylene hydrazides and compared with comventional method. Spectral properties and mass spectral fragmentation pattern of these hydrazides \& hydrazones were also discussed. It has been found that some of these hydrazides have attractant activity and some have repellent activity depending on their concentration against mosquitoes and we will be published somewhere elsc. ${ }^{14}$

\section{EXPERIMENTAL}

\section{General}

All the reagents and solvent were obtained from commercial sources and used without further purification. Velting points were taken in open capillary in an electro themal poinl apparatus and are uncorrected. IR spectra were recorded as $\mathrm{KBr}$ pellet using Perkin I:Imer Spectrum BX J [-IR spectrophotoneter, ' $\mathrm{H}$ NMR spectra in $\mathrm{CI} \mathrm{Cl}_{3} / \mathrm{D}$ MSO-d, on a Bruker Avance $(400 \mathrm{MHz})$ spectrometer using TMS as internal reference. ${ }^{1} \mathrm{C}$ NMR spectra were recorded on the same instrument at $100 \mathrm{MHz}$ using the same solvent and intemal reference. Chemical 
shift is reported in $\delta$ (PPM). Mass spectra were recorded on a Finnigan matt TSQ 700 mass spectrometer. The progress of all reactions was monitored by TLC on $2.0 \times 5.0-\mathrm{cm}$ aluminum sheets precoated with silica gel to a thickness of $0.25-\mathrm{mm}$ (Merck). Microwave assisted syntheses were carried out in Samsung CK 138 F domestic microwave oven.

\section{Dodecanoic acid hydrazide 2 a}

Methyl dodecanoate (10 mmol) was dissolved in $99 \%$ hydrazine hydrate (10 $\mathrm{mmol})$ in a conical tlask and was placed in donestic microwave overn. After irradiation at 180 Watt for 10 minutes, the solid product was washed with the ethanol to give dodecanoic acid hydrazide $2 \mathrm{a}$ ( $85 \%$ yield) (Table 1 \& 2)

General procedure for the synthesis of long chain aliphatic acid (2-hydroxy benzylidene) hydrazides \& (1H-indol-3-ylmethylene) hydrazides

Conventional synthesis

To a stirred solution of carboxylic acid hydrazide (5 mmol) in ethanol was added aldehyde $(5 \mathrm{mmol}$ ) and the reaction mixture was refluxed for 3-4 hrs. After completion of the reaction, solvent was evaporated \& solid product was separated, filtered, dried, \& recrystallized from ethanol.

\section{Microwave assisted synthesis}

Carboxylic acid hydrazide $(5 \mathrm{mmol})$ was mixed with aldehyde $(5 \mathrm{mmol})$ in a conical flask and the mixture was irradiated with microwave irradiation in microwave oven for specified period (300 Watt). The reaction was monitored by TLC. After completion of the reaction, the solid product was recrystallized from ethanol to give the pure substituted hydrazides (hydrazones).

Dodecanoic acid (2-hydroxy benzylidene) hydrazide $3 a$.

$\mathrm{Mp}: 108-110^{\circ} \mathrm{C}, \mathbb{R}(\mathrm{KBr}): 3432,3238,2922,1670$, $1560,1525,1380,758 \mathrm{~cm}^{-1}$. ${ }^{1} \mathrm{H}$ NMR $(400 \mathrm{MHz}$, DMSO-d $\left.\mathrm{d}_{6}\right) \delta=10.8(\mathrm{~s}, \mathrm{lH}, \mathrm{OH}), 8.5(\mathrm{~s}, \mathrm{lH}, \mathrm{CONH}$, exchangeable), $8.0(\mathrm{~s}, 1 \mathrm{H}, \mathrm{N}=\mathrm{CH}), 6.9-7.5(\mathrm{~m}, 4 \mathrm{H}$, Ar-H), $0.9\left(\mathrm{t}, 3 \mathrm{H}, \mathrm{CH}_{3}\right), 1.2$ (conglomerate, $16 \mathrm{H}$, $\left.\mathrm{CH}_{2}\right), 1.4\left(\mathrm{p}, 2 \mathrm{H}, \mathrm{CH}_{2}\right), 2.5$ (t, $\left.2 \mathrm{H}, \mathrm{CH}_{2} \mathrm{CO}\right), \mathrm{MS}: \mathrm{m} / \mathrm{z}$ (\%) 318 (40), [ $\left.\mathrm{C}_{19} \mathrm{H}_{3 \mu} \mathrm{N}_{2} \mathrm{O}_{2}\right]^{+}, 275$ (12), 219 (10), 191
$(14), 178(25), 136(100), 135(79), 119(71), 107(47)$, $93(16), 91(30), 65(26)$.

Dodecanoic acid (1H-indol-3-ylmethylene) hydrazide $\mathbf{3 b}$.

Mp: $145-147^{\circ} \mathrm{C}, \mathrm{IR}(\mathrm{KBr}): 3245,3055,2924$, $1638,1597,1443,1246,992,740 \mathrm{~cm}^{-1}$. ${ }^{1} \mathrm{H}$ NMR $\left(400 \mathrm{MHz}, \mathrm{DMSO}-\mathrm{d}_{6}\right) \delta=10.1(\mathrm{~s}, \mathrm{lH}, \mathrm{NH}$ indole, exchangeable), 8.8 (s, $1 \mathbf{H}, \mathrm{CONH}$, exchangeable), $8.5(\mathrm{~s}, 1 \mathrm{H}, \mathrm{N}=\mathrm{CH}), 7.0-7.8(\mathrm{n}, 4 \mathrm{x}$ Ar-H, $1 \mathrm{x}$ indole $\mathrm{CH}), 2.1\left(\mathrm{t}, 2 \mathrm{H}, \mathrm{CH}_{2} \mathrm{CO}\right) 1.6\left(\mathrm{p}, 2 \mathrm{H}, \mathrm{CH}_{2}\right), 1.2$ (conglomerate, $16 \mathrm{H}, \mathrm{CH}_{2}$ ) $1.0\left(\mathrm{t}, 3 \mathrm{H}, \mathrm{CH}_{3}\right.$ ) MS: $\mathrm{m} / 2$ (\%) $341(41),\left[\mathrm{C}_{71} \mathrm{H}_{72} \mathrm{~N}_{3} \mathrm{O}\right]^{-}, 228(45), 201$ (13), $186(5)$, 185 (4), 184 (6), 159 (48), 158 (4), 157 (15), 155 (4), 142 (100), $130(25), 116(24), 114$ (14), 59 (38).

Tetradecanoic acid (2-hydroxy benzylidene) hydrazide $3 \mathrm{c}$.

Mp: $113-115^{\circ} \mathrm{C}, \mathrm{RR}(\mathrm{KBr}): 3447,3138,2920,1670$, $1567,1491,1390,753 \mathrm{~cm}^{-1}$. 'H NMR $(400 \mathrm{MHz}$, DMSO-d $\left.\mathrm{d}_{6}\right) \delta=10.5(\mathrm{~s}, 1 \mathrm{H}, \mathrm{OH}), 8.4(\mathrm{~s}, 1 \mathrm{H}, \mathrm{CONH}$, exchangeable), $8.1(\mathrm{~s}, 1 \mathrm{H}, \mathrm{N}=\mathrm{CH}), 7.1-7.9(\mathrm{~m}, 4 \mathrm{H}$, $\mathrm{Ar}-\mathrm{H}$ ), $1.1\left(\mathrm{t}, 3 \mathrm{H}, \mathrm{CH}_{3}\right), 1.5$ (conglomerate, $20 \mathrm{H}$, $\left.\mathrm{CH}_{2}\right) 1.9\left(\mathrm{p}, 2 \mathrm{H}, \mathrm{CH}_{2}\right), 2.2\left(\mathrm{t}, 2 \mathrm{H}, \mathrm{CH}_{2} \mathrm{CO}\right), \mathrm{MS}: \mathrm{n} / 2$ (\%) $346(16),\left[\mathrm{C}_{21} \mathrm{H}_{34} \mathrm{~N}_{2} \mathrm{O}_{2}\right]^{+}, 219(8), 233$ (4), 219 (5), $205(4), 191(10), 178(15), 177(4), 163(6), 136(100)$, 135 (79), $120(65), 119(57), 107(34), 99(7), 93(13)$, $91(30), 71(45), 65(26), 59(100), 43(73)$.

Tetradecanoic acid(1H-indol-3-ylmethylene) hydrazide $3 \mathrm{~d}$.

Mp: 154-156 ${ }^{\circ} \mathrm{C}, \mathrm{RR}(\mathrm{KBr}): 3210,3035,2923,1642$, $1447,1357,1246,995,744 \mathrm{cn}^{-1} .{ }^{1} \mathrm{H}$ NMR $(400 \mathrm{MHz}$, DMSO-d $\left.\mathrm{d}_{6}\right) \delta=10.0(\mathrm{~s}, \mathrm{lH}, \mathrm{NH}$ indole, exchangeable), $8.4(\mathrm{~s}, 1 \mathrm{H}, \mathrm{CONH}$, exchangeable), $8.0(\mathrm{~s}, 1 \mathrm{H}$, $\mathrm{N}=\mathrm{CH}), 7.2-7.9(\mathrm{~m}, 4 \times \mathrm{Ar}-\mathrm{H}, 1 \times$ indole $\mathrm{CH}), 2.3$ (t, 2H, $\left.\mathrm{CH}_{2} \mathrm{CO}\right), 1.9\left(\mathrm{p}, 2 \mathrm{H}, \mathrm{CH}_{2}\right), 1.4$ (conglomerate, $\left.20 \mathrm{H}, \mathrm{CH}_{2}\right), 0.9\left(\mathrm{t}, 3 \mathrm{H}, \mathrm{CH}_{3}\right) \mathrm{MS}: \mathrm{n} / 2(\%) 369(54)$, $\left[\mathrm{C}_{23} \mathrm{H}_{35} \mathrm{~N}_{3} \mathrm{O}\right]^{+}, 228(43), 211(4), 201(5), 186(5)$, 185 (4), 184 (6), 159 (74), 158 (14), 157 (15), 155 (4), $142(100), 130(25), 116(20), 114(14), 59(40)$.

Hexadecanoic acid (2-hydroxy benzylidene) hydrazide 3 e.

Mp: $118-120^{\circ} \mathrm{C}$, IR (KBr): $3440,3038,2921$, 1670, 1560, 1466, 1278, $752 \mathrm{~cm}^{-1}$, 'H NMR $(400 \mathrm{MHz}$, DMSO-d $\left.\mathrm{d}_{6}\right) \delta=10.3(\mathrm{~s}, 1 \mathrm{H}, \mathrm{OH}), 8.8(\mathrm{~s}, \mathrm{lH}, \mathrm{CONH}$, exchangeable), $8.5\left(\mathrm{~s}, 1 \mathrm{H}, \mathrm{N}^{-}=\mathrm{CH}\right), 7.0-7.8(\mathrm{~m}, 4 \mathrm{H}$, $\mathrm{Ar}-\mathrm{H}), 0.9\left(\mathrm{t}, 3 \mathrm{H}, \mathrm{CH}_{\dot{y}}\right), 1.3$ (conglomerate, $24 \mathrm{H}$, 
$\left.\mathrm{CH}_{2}\right), 1.5$ (p, $\left.2 \mathrm{H}, \mathrm{H}_{2}\right), 2.1$ (t, $\left.2 \mathrm{H}, \mathrm{CH}_{2} \mathrm{CO}\right), \mathrm{MS}: \mathrm{m} / \mathrm{z}(\%)$ 374 (26), $\left[\mathrm{C}_{33} \mathrm{H}_{38} \mathrm{~N}_{2} \mathrm{O}_{3}\right]^{-}, 345$ (3), 331 (4), 317 (4), 303 (3), $289(3), 247$ (4), $219(5), 191$ (10), $179(5)$, $178(25), 163(6), 136(100), 120(65), 119(57)$, $107(34), 99(11), 93(10), 91(20), 71(25), 65(16)$, $59(50), 43(30)$.

Hexadecanoic acid(1H-indol-3-ylmethylene) hydrazide $3 f$.

Mp: $160-162^{\circ} \mathrm{C}, \mathbb{R}(\mathrm{KBt}): 3227,3055,2916,1638$, $1617,1361,1247,991,739 \mathrm{~cm}^{-1} .{ }^{1} \mathrm{H}$ NMR $(400 \mathrm{MHz}$, DMSO-d $\left.\mathrm{d}_{6}\right) \delta=10.2(\mathrm{~s}, 1 \mathrm{H}, \mathrm{NH}$ indole, exchangeable), $8.5(\mathrm{~s}, 1 \mathrm{H}, \mathrm{CONH}$, exchangeable), $8.0(\mathrm{~s}, 1 \mathrm{H}$, $\mathrm{N}=\mathrm{CH}$ ), 7.0-7.8 (m, $4 \times$ Ar- $\mathrm{H}, 1 \times$ indole $\mathrm{CH}$ ), 2.1 (t, $2 \mathrm{H}, \mathrm{CH}_{2} \mathrm{CO}$ ), $1.5\left(\mathrm{p}, 2 \mathrm{H}, \mathrm{CH}_{7}\right), 1.2$ (conglomerate, $\left.20 \mathrm{H}, \mathrm{CH}_{2}\right), 0.9\left(\mathrm{t}, 3 \mathrm{H}, \mathrm{CH}_{3}\right) \mathrm{MS}: \mathrm{m} / \mathrm{z}(\%) 397(45)$, $\left[\mathrm{C}_{23} \mathrm{H}_{34} \mathrm{~N}_{3} \mathrm{O}\right]^{+}, 368$ (4), 354 (3), 340 (3), 298 (3), 284 (4), $256(45), 228$ (4), 201(10), 185 (4), $184(6)$, $159(70), 158$ (24), $157(12), 155(4), 142(100)$, $130(23), 116(20), 114(14), 59(54)$.

Acknowledgements. Authors sincerely thank Er K Sekhar, Director, Defence Research and Development Establishment, Gwalior for keen interest and encouragement. We are also thankful to Dr. D.K. Dubey for his valuable suggestions \& comments and to Dr. Semwal for recording spectra.

\section{REFERENCES}

1. Bonicke, R; Krach, J. Z. Hig Infektionalfonch 1954, 139, 140 .

2. Haksar, C. N.; Malhotra, R. C.; Pandya G; Sethi, R. K.
Lab. J. Sc. Technol., 1981, $9 B, 34$

3. Binon, F.; Royer, R. J. Chem. Soc., 1953, 1358.

4. Zikolova, Sv. Farmatoiya, 1965, 15(4), 185.

5. Roman, K. L. Span. Patent, ES 506,945, 1982 (Chem. Abstr, 1985, 89, 67124).

6. Dhadialla, T. S.; Carlson, G R; Le, D. P. Annual Revient of Entonolog, 1998, 43, 545-569.

7. Banik, B. K.; Barakat, K. J.; Wagle, W. R; Manhas, M. S.; Bose, A. K. J. Org. Chem., 1999, 64, 5746

8. Ganesan K.; Mendki, M. J.; Suyauarayana, M. V. S.; Prakash, S.; Malhotra, R C. Australion Journal of Entonologv, 2000, 45, 75-80.

9. Mendki, M. J.; Ganesan, K.; Prakash, S.; Sunyanarayana, M. V. S.; Mall rota, R C.: Rao, K M:- Vaidyanathaswany, R. Current Science, 2000, 78, 1295-1296.

10. Bentley, M. D.; McDaniel, I. N.; Yatagai, M.; Lee, H. P.; Maynard, R. Environmental Entomologr, 1979, 8, 206-209.

11. Knight, J. C.; Corbet, S. A. Jommal of Anterican Wosquito Control Association, 1991, 7, 37-41.

12. Villemine, D.; Martin, B. J. Chen. Res., 1994, 146.

13. Lu, Y. F.; Fallis, A. G. Con. J. Chem., 1995, 73, 2239.

14. Soriente, A.; Spinella, A.; DeRosa, M.; Giordano, M.; Seettri, A. Tetwahedron Lett, 1997, 38, 289.

15. Suarez, M.; Loupy, A.; Salfran, E.; Moran, L.; Rolando, E. Hererocycles, 1999, 51, 21 .

16. Goncalo, P.; Roussel, C.; Melot, I. M.; Vetrel, J. J. Chem. Soc. Perkin Trams 2, 1999, 2111.

17. Danks, T. N. Tetrahedron Lett, 1999, 40, 3957.

18. Verma, R. S. Green Chemisnv, 1999, 1, 43.

19. Rishiswar, P.; Awasthi, S.; Jain, N.; Prakash, S.; Ganesar, K.; Malhotra, RC.; Vịayarashavan, R Oripositional responses of Aedes aegypti (Diptera: Culicidae) to novel synthesized lones chain aliphatic hydrazides (to be communicated to Vector Ecologv). 